Национальный медицинский исследовательский центр эндокринологии, Москва, Российская Федерация

Онкоэндокринология - новый вектор развития персонализированной медицины, строящийся на научнотворческой коммуникации эндокринологов, онкологов, морфологов, радиологов, генетиков, медицинских физиков, биологов, химиков, математиков.

В журнале «Проблемы эндокринологии» решением редакционной коллегии введен раздел «Онкоэндокринология». В нем планируется публиковать обзорные и оригинальные статьи по вышеозначенным научно-прикладным направлениям. Приглашаем всех заинтересованных авторов и читателей к сотрудничеству.

КЛЮЧЕВЫЕ СЛОВА: онкоэндокринология, онкология, эндокринология.

\title{
ONCOENDOCRINOLOGY — THE INNOVATIVE INTERDISCIPLINARY BASE FOR PERSONALIZED MEDICINE
}

\author{
(C) Pavel O. Rumyantsev*
}

Endocrinology Research Centre, Moscow, Russian Federation

Oncoendocrinology is a new vector in personalized medicine based on the scientific and creative communication of endocrinologists, oncologists, morphologists, radiologists, geneticists, medical physicists, biologists, chemists, and mathematicians. A section «Oncoendocrinology» has been introduced in the Problems of Endocrinology journal by the editorial board. It is planned to publish reviews and original articles in the aforementioned scientific and applied fields. We invite all interested authors and readers to cooperation.

KEYWORDS: oncoendocrinology, oncology, endocrinology.

Новообразования гормонсекретирующих органов и клеточных систем, а также гормонзависимые карциномы имеют существенные отличия от других опухолей из-за особенностей биологической природы и клинического течения, требующих углубленного изучения и совершенствования алгоритмов диагностики и лечения.

В фокусе онкоэндокринологии - трансляционные исследования, диагностика, лечение, прогноз и реабилитация пациентов с:

- нейроэндокринными опухолями различной локализации (желудочно-кишечный тракт, легкие и пр.);

- опухолями эндокринных органов - щитовидной железы, паращитовидных желез, поджелудочной железы, надпочечников, гипофиза;

- хромаффинными опухолями (феохромоцитома, параганглиома, нейробластома);

- гормонзависимыми карциномами молочной железы, предстательной железы и пр.;

- эндокринными осложнениями противоопухолевой терапии.

Нейроэндокринные опухоли - гетерогенная группа новообразований различной локализации - составляют от 1 до 2\% всех опухолей; встречаются в клинической практике в структуре злокачественных новообразований желудочно-кишечного тракта, легких, кожи (опухоль Меркеля), молочной железы, предстательной железы, других органов и тканей. Распознать их нередко сложно, иногда трудности возникают даже при гистологическом исследовании. К нейроэндокринным опухолям также относят опухоли гипофиза, медуллярный рак щитовидной железы, опухоли паращитовидных желез, надпочечников, поджелудочной железы.

Различают гормонсекретирующие ( 20\%) и гормонально-неактивные ( 80\%) нейроэндокринные опухоли.

В сравнении с другими злокачественными новообразованиями (легких, прямой кишки и др.), достигших своего плато в течение последних десятилетий, заболеваемость нейроэндокринными опухолями прогрессивно росла и продолжает увеличиваться, составляя 1 случай в 1970-х годах, 7 - в 2012 г., 10 на 100 тыс. населения в 2018 г. Так, среди новообразований поджелудочной железы нейроэндокринные опухоли составляют до 7\%. В поджелудочной железе могут развиваться и гормонально-активные нейроэндокринные опухоли - инсулиномы, гастриномы и пр.

Онкоэндокринология - междисциплинарное направление на стыке онкологии и эндокринологии, радиологии и генетики. В структуре ФГБУ «НМИЦ эндокринологии» Минздрава России в 2018 г. был создан Институт онкоэндокринологии для углубленного изучения опухолей эндокринной природы и совершенствования лечебно-диагностических и реабилитационных 
подходов с использованием передовых молекулярногенетических технологий, мультимодальной визуализации и радиотераностики. Институт онкоэндокринологии задуман для развития следующих перспективных направлений персонализированной медицины.

1. Радионуклидная молекулярная визуализация и радиомика: однофотонная эмиссионная томография, сопряженная с компьютерной томографией, а в перспективе - совмещенная с рентгеновской и магнитно-резонансной - позитронно-эмиссионная томография.

Молекулярная визуализация с помощью однофотонной эмиссионной томографии, сопряженной с компьютерной томографией выполняется при заболеваниях щитовиднойжелезы, паращитовидныхжелез, нейроэндокринных и хромаффинных опухолях. В отделении радионуклидной диагностики Института онкоэндокринологии выполняются также перфузионная кардиосцинтиграфия (в покое и с нагрузкой), нефросцинтиграфия, остеосцинтиграфия и пневмосцинтиграфия.

2. Радиотераностика: различные виды радионуклидной терапии - радиометаболическая, пептидрецепторная (радиолигандная), радиоиммунная.

В отделении радионуклидной терапии выполняются радиойодтерапия рака щитовидной железы и тиреотоксикоза, терапия костных метастазов рака предстательной железы. Планируется развитие перспективных направлений радиотераностики нейроэндокринных (177Lu-DOTA-TATE/TOC/NOC) и хромаффинных опухолей (MIBG-131), рака простаты (177Lu-PSMA) и других.

3. Дозиметрическое обеспечение радиотераностики.

Персонализированная радиотераностика требует совершенствования дозиметрии пациентов и биодозиметрии крови на этапах диагностики с целью планирования максимально эффективной и безопасной радионуклидной терапии.

4. Молекулярная онкоэндокринология (радиогеномика).

Лаборатория молекулярной онкоэндокринологии находится в стадии создания. Ее главной задачей будет поиск диагностических и прогностических биомаркеров для улучшения диагностики и лечения опухолей эндокринной природы. Также актуально изучение наследственно-генетических основ возникновения и особенностей клинического течения синдромов множественной эндокринной неоплазии. Развитие прикладных трансляционных молекулярно-генетических исследований в области онкогеномики и радиогеномики позволит персонализировать тактику диагностики и лечения пациентов.

Определение генетических характеристик опухоли основательно вошло в клиническую практику как в диагностических, так и в прогностических целях, например, при выборе таргетной терапии. В последние годы активно развивается направление, позволяющее получить количественные характеристики опухоли на основании анализа изображения, полученного с использованием различных неинвазивных методов визуализации (ультразвуковое исследование, компьютерная томография, магнитно-резонансная томография, однофотонная эмиссионная и позитронная эмиссионная томография), - радиомика. Следующим шагом в получении наиболее исчерпывающей информации об опухоли является совмещение ее молекулярно-генетического и метаболического профиля с помощью современных «омиксных» технологий с молекулярной визуализацией опухоли радиогеномика.

Для различных злокачественных новообразований эндокринной природы планируется определять клинически значимые герминальные и соматические мутации. Например, в случае рака щитовидной железы наиболее часто встречаются мутации в генах BRAF, TERT, RET/PTC, RAS, PIK3CA, PAX8/PPARG, EGFR, CTNNB1, TP53. Для пациентов с феохромоцитомой/параганглиомой показано проведение генетического анализа мутаций $S D H x, V H L$, RET, NF1, MAX, TMEM127 при множественной эндокринной неоплазии 1-го типа - мутаций MEN1, а 2-го типа гена $R E T$.

B последние годы большое внимание уделяется возможности выявления опухолевой ДНК в крови пациентов, так называемой жидкостной биопсии. Важным приложением жидкостной биопсии является неинвазивный и чувствительный мониторинг ответа на проводимую терапию, а также рецидива опухоли. Жидкостная биопсия - поиск в крови циркулирующих опухолевых клеток, фрагментов их ДНК, везикул и т.п. В случае рецидива рака щитовидной железы в крови могут быть определены циркулирующие опухолевые ДНК, в том числе с мутацией $B R A F^{\mathrm{V} 600 \mathrm{E}}$. При этом концентрация циркулирующих опухолевых ДНК коррелирует со стадией заболевания, а их присутствие с мутацией $B R A F^{V 600 E}$ свидетельствует о вероятной радиойодрезистентности опухоли. Циркулирующие опухолевые ДНК зачастую выявляются в плазме крови раньше, чем опухоль может быть выявлена другими методами.

5. Интраоперационная визуализация: нейромониторинг, гамма-зондирование, флуоресцентная и оптическая спектрометрия).

Внедрение инновационных методов интраоперационной визуализации, с одной стороны, позволяет осуществлять прецизионное хирургическое удаление патологических образований, а с другой - сохранять важнейшие функциональные структуры (например, паращитовидные железы при операциях на щитовидной железе, гортанные нервы), что обеспечивает повышение радикальности операции и снижение риска послеоперационных осложнений. Вместе с интраоперационным нейромониторингом, позволяющим осуществлять функциональную визуализацию двигательных нервов и их ветвей во время операции, это повышает радикальность и безопасность хирургического лечения, что крайне актуально при выполнении открытых и эндоскопических операций. Внедрение инновационных методов интраоперационной визуализации двигательных нервов, кровоснабжения, функциональной активности эндокринных желез, метаболизма опухоли, поиск сторожевых лимфоузлов позволит хирургу увидеть in vivo патологический процесс и предохранить функционально важные структуры от повреждения. В перспективе планируется внедрение и развитие методов многоканального гамма-зондирования, аутофлуоресцентной и оптической спектрометрии, а также их комбинации. 


\section{6. Нейрофониатрическая реабилитация}

Нередко осложнением операции на щитовидной железе становится нарушение голоса, глотания, дыхания. Нами уже накоплен опыт эффективной фониатрической реабилитации, с помощью которой удается значительно улучшить голос, дыхание и глотание после осложненных операций в проекции щитовидной железы.

Планируется не только совершенствование данного направления, но и внедрение методов эндоскопической хирургической реабилитации пациентов, имеющих серьезные проблемы с дыханием вследствие двустороннего паралича гортани.

\section{7. Междисциплинарный банк данных}

Направление имеет целью создание условий на основе информационных технологий для улучшения доказательной базы ретроспективных многоцентровых клинических исследований. Уже реализован междисциплинарный банк данных по медуллярному раку щитовидной железы и синдрому множественной эндокринной неоплазии второго типа. В настоящее время нами ведется опытно-конструкторская работа по созданию междисциплинарного банка данных по дифференцированному раку щитовидной железы, резистентного к терапии радиоактивным йодом.

\section{ИНФОРМАЦИЯ ОБ АВТОРАХ [AUTHORS INFO]}

*Румянцев Павел Олегович, д.м.н. [Pavel O. Rumyantsev, MD, PhD]; адрес: Россия, 117036, ул. Дмитрия Ульянова, д. 11, к. 2 [address: 11 k. 2 Dmitry Ulyanova street, 117036 Moscow, Russia]; e-mail: pavelrum@gmail.com, eLibrary SPIN: 7085-7976, ORCID: https://orcid.org/0000-0002-7721-634X

\section{ЦИТИРОВАТЬ:}

Румянцев П.О. Онкоэндокринология - инновационная междисциплинарная платформа персонализированной медицины. - 2020. - Т. 66. - №1. - C. 4-6. doi: https://doi.org/10.14341/probl12414

\section{TO CITE THIS ARTICLE:}

Rumyantsev PO. Oncoendocrinology - the Innovative Interdisciplinary Base for Personalized Medicine. Problems of Endocrinology. 2020;66(1):4-6. doi: https://doi.org/10.14341/probl12414 